\title{
Depth-averaged momentum equation for gravity currents with varying density: coefficient in pressure term
}

\section{Dubravka Pokrajac, Sara Venuleo \& Mário J. Franca}

To cite this article: Dubravka Pokrajac, Sara Venuleo \& Mário J. Franca (2018) Depth-averaged momentum equation for gravity currents with varying density: coefficient in pressure term, Journal of Hydraulic Research, 56:3, 424-430, DOI: 10.1080/00221686.2017.1335245

To link to this article: https://doi.org/10.1080/00221686.2017.1335245

曲 Published online: 31 Jul 2017.

Submit your article to this journal $\sqsubset$

Џلll Article views: 178

Q View related articles $\square$

View Crossmark data 〔 
Technical note

\title{
Depth-averaged momentum equation for gravity currents with varying density: coefficient in pressure term
}

\author{
DUBRAVKA POKRAJAC, Professor, School of Engineering, University of Aberdeen, Aberdeen, UK \\ Email:d.pokrajac@abdn.ac.uk (author for correspondence)
}

SARA VENULEO, PhD student, Laboratory of Hydraulic Constructions, École polytechnique fédérale de Lausanne, Lausanne, Switzerland

MÁRIO J. FRANCA, Research and Teaching Associate, Laboratory of Hydraulic Constructions, École polytechnique fédérale de Lausanne, Lausanne, Switzerland

\begin{abstract}
Gravity currents are often modelled by means of shallow water equations (SWEs). In these models, simplifications such as the consideration of a constant layer-averaged density are common. This note presents the complete and general derivation of a $2 \mathrm{D}$ depth-averaged momentum equation for gravity currents with density and velocity varying in the bed-normal direction. Special attention is given to the pressure term which is evaluated for constant, linear and exponential density profile. The shape of the density profile has implications for the momentum balance: the assumption of constant density leads to an overestimation of the driving force due to pressure gradient by a factor of $33 \%$ for linear density profile and up to $50 \%$ for an exponential profile. It also leads to an overestimation of celerity in numerical models based on traditional SWEs by factor of $22 \%$ and around $40 \%$ for linear end exponential density profiles respectively.
\end{abstract}

Keywords: Gravity currents; pressure term; shallow water equations; varying density; reduced gravity; celerity

\section{Introduction}

Gravity currents are geophysical flows driven by density difference between two fluids caused by gradients in temperature, dissolved substances or particles in suspension. Velocity and density profiles typical for gravity currents are often nonuniform in the bed-normal direction, as reported by several experimental and numerical studies (Altinakar et al., 1996; Kneller et al., 1999; Parker et al., 1987; Sequeiros et al., 2010; Stagnaro \& Bolla, 2014; Ottolenghi et al., 2016a, 2016b). Traditionally the depth-varying shape of the profiles is taken into account through multiplicative factors, often called shape factors, which appear in the shallow-water layer-integrated equations (SWEs) (Chu et al., 1979; Hogg \& Pritchard, 2004; Parker et al., 1987; Sequeiros et al., 2010). In this note we refer to these multiplicative factors simply as coefficients. Particular coefficients are named after the SWE term where they appear, e.g. momentum coefficient and pressure coefficient. A coefficient in an SWE term is defined as the ratio of the value of the term obtained by integration over the current depth and the same term obtained from depth-averaged quantities.

The values of SWE coefficients/shape factors have been reported for some flow regimes and bed roughness (Parker et al., 1987; Sequeiros et al., 2010). Some difficulties in comparing these coefficients are associated with the definition of the current height, $h$ (Stacey \& Bowen, 1988). According to the definitions of Altinakar et al. (1996) and of Ellison and Turner (1959), current height is notional, and all depth-averaged quantities are found by integration between the bed level and infinity. On the other hand, Chu et al. (1979) use a physicallybased current depth for expressing SWE coefficients/shape factors, but these are subsequently set to one. Although the nonuniformity of the density and velocity profiles in gravity currents has been well established, SWE models often set coefficients in all terms to the value that corresponds to uniform profiles, i.e. to unity (Chu et al., 1979; Stacey \& Bowen, 1988; Ungarish, 2009; Adduce et al., 2012; Lombardi et al., 2015). This may not be justified in some cases, for example for supercritical flows (Sequeiros et al., 2010).

Received 11 July 2016; accepted 23 May 2017/Open for discussion until 31 December 2018. 
This note aims to provide the basis for estimating various SWE coefficients. It therefore presents a rigorous derivation of 2D depth-averaged momentum equations for gravity currents, following a procedure similar to Pokrajac and Kikkert (2011). Derivation of other balance equations such as volume and mass balance are analogous, and they have been omitted for brevity. Depth averaging is performed until the top boundary of the current which must be physically-based defined. Various options for defining this boundary are beyond the scope of the note.

The note is focused solely on the coefficient which appears in the pressure term due to the bed-normal variation of density. It will be shown that this coefficient, termed pressure coefficient, can be easily evaluated for typical density profiles from the literature and incorporated in the existing SWE simulation models. It will also be shown that, for cases with substantial density variation across current depth, omitting pressure coefficient in SWEs results in significant error. Other SWE coefficients require a much more elaborate analysis before they can be evaluated and incorporated in simulations models if/where necessary. These coefficients are therefore also beyond the scope of the note.

\section{Integral form of the momentum balance equation}

\subsection{Definitions}

The definition sketch with the main variables is shown in Fig. 1. We consider a density current propagating over a flat bed, which may be inclined in both longitudinal and lateral directions - corresponding angles are $\alpha$ and $\beta$, respectively. A right-handed Cartesian coordinate system used throughout the note consists of a longitudinal coordinate $x$, lateral coordinate $y$ and bed-normal coordinate $z$ with the origin $z=0$ at the bed. The corresponding components of current velocity are $u, v, w$, and those of the velocity of the interfaces are $U, V, W$. An alternative coordinate system $x_{i}, i=1,2,3(\equiv x, y, z)$ with corresponding velocity components $u_{i}, U_{i}$ is also used wherever it produces simpler expressions, and in such case Einstein summation convention applies.
Geometry of the control volume is defined in a local coordinate system $(\xi, \eta, z)$ : the stream-wise extent of the domain is $-\Delta x / 2 \leq \xi \leq \Delta x / 2$, and its lateral extent is $-\Delta y / 2 \leq \eta \leq$ $\Delta y / 2$. In the bed-normal direction, $z$, the control volume covers the entire current depth until the interface with the ambient fluid ( $S_{\mathrm{I}}$ in Fig. 1), i.e. $0 \leq z \leq z_{\mathrm{I}}=h$. The ambient fluid is assumed to be stagnant and to have a constant density, $\rho_{0}$. For the purpose of deriving depth-averaged momentum balance equation, the average of a general fluid variable $\psi$ over the current depth is defined as:

$$
\langle\psi\rangle_{h}=\frac{1}{h} \int_{0}^{z_{\mathrm{I}}} \psi \mathrm{d} z
$$

\subsection{Momentum balance in terms of relative pressure and density difference}

We start from the differential form of the momentum balance equation for an incompressible fluid with generally variable density $\rho$ :

$$
\frac{\partial \rho u_{j}}{\partial t}+\frac{\partial \rho u_{j} u_{i}}{\partial x_{i}}=\rho g_{j}-\frac{\partial p}{\partial x_{j}}+\frac{\partial \tau_{i j}}{\partial x_{i}}, \quad i, j=1,2,3
$$

For the stagnant ambient fluid the momentum equation reduces to:

$$
\frac{\partial p_{0}}{\partial x_{j}}=\rho_{0} g_{j}
$$

Combining the previous two equations yields:

$$
\frac{\partial \rho u_{j}}{\partial t}+\frac{\partial \rho u_{j} u_{i}}{\partial x_{i}}=g_{j}\left(\rho-\rho_{0}\right)-\frac{\partial\left(p-p_{0}\right)}{\partial x_{j}}+\frac{\partial \tau_{i j}}{\partial x_{i}}
$$

where $p_{0}$ is the pressure of ambient fluid at any point as it would be without the presence of the density current. This way of expressing momentum balance makes the derivation of the depth-averaged equation somewhat simpler. Integration of Eq. (3) over an arbitrary control volume $\forall$, enclosed within a (a)

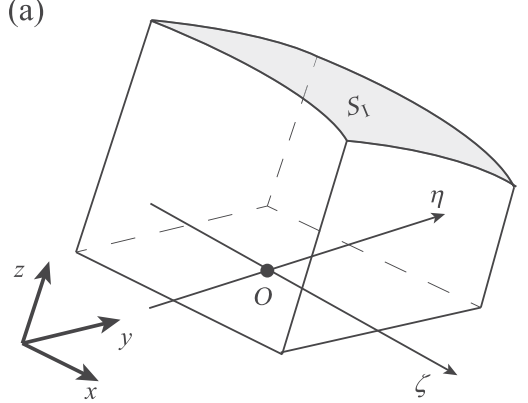

(b)

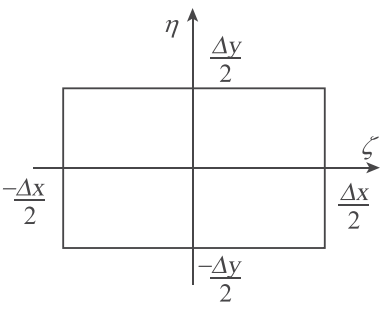

(c)

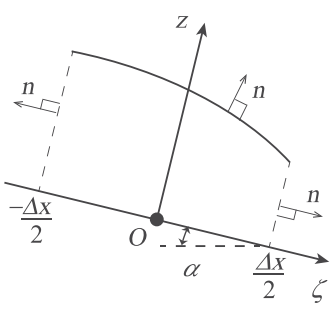

(d)

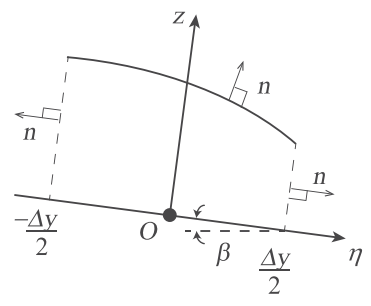

Figure 1 Sketch with the definition of the control volume and coordinate systems: (a) control volume; (b) plan view of the control volume; (c) longitudinal domain; (d) lateral domain 
surface $S$ which moves at velocity $\vartheta_{i}$, yields:

$$
\begin{gathered}
\underbrace{\int_{\forall} \frac{\partial \rho u_{j}}{\partial t} \mathrm{~d} \forall+\int_{S} \rho u_{j} \vartheta_{i} n_{i} \mathrm{~d} S+\int_{S} \rho u_{j}\left(u_{i}-\vartheta_{i}\right) n_{i} \mathrm{~d} S}_{R M} \\
=\underbrace{\int_{\forall}\left(\rho-\rho_{0}\right) g_{j} \mathrm{~d} \forall}_{G}-\underbrace{\int_{S}\left(p-p_{0}\right) n_{j} \mathrm{~d} S}_{P} \\
+\underbrace{\int_{S} \tau_{i j} n_{i} \mathrm{~d} S}_{T}
\end{gathered}
$$

The terms on the left hand side of Eq. (4) represent, respectively, the rate of change of momentum within the control volume, the momentum flux through the surface $S$ due to its own movement, and the momentum flux due to the movement of the fluid relative to $S$. Collectively these terms also represent the Rate of change of $M$ omentum (in a control volume moving with the fluid), so they are denoted with $R M$. The terms on the right hand side represent the net force acting on the control volume due to Gravity, Pressure and bed shear sTress, respectively, so they are denoted, in the same order, with $G, P$, and $T$. In the next subsections terms $R M, G, T$ and $P$ are developed for the control volume shown in Fig. 1, and for the $x$-momentum. Derivation of the balance equation for the $y$-momentum is analogous.

\subsection{Rate of change of momentum terms RM}

The left hand side of Eq. (4) is now expressed for the control volume shown in Fig. 1, contained within a surface which consists of the bottom, B, the four bed-normal faces, and the interface between the current and the ambient fluid, I. This interface moves at velocity $U_{i}$, whereas all other surfaces are stationary. Furthermore, the bottom surface is considered solid so that no-slip condition applies and all velocity components along this surface are zero. The left hand side of Eq. (4) therefore becomes:

$$
\begin{aligned}
R M= & \underbrace{\int_{-\Delta x / 2}^{\Delta x / 2} \int_{-\Delta y / 2}^{\Delta y / 2} \int_{0}^{z_{\mathrm{I}}(\xi, \eta)} \frac{\partial \rho u}{\partial t} \mathrm{~d} z \mathrm{~d} \eta \mathrm{d} \xi}_{\text {Term } 1}+\underbrace{\int_{S_{\mathrm{I}}} \rho u U_{i} n_{i} \mathrm{~d} S_{\mathrm{I}}}_{\text {Term } 2} \\
& +\underbrace{\int_{S_{\mathrm{I}}} \rho u\left(u_{i}-U_{i}\right) n_{i} \mathrm{~d} S_{\mathrm{I}}}_{\text {Term } 3}-\int_{-\Delta y / 2}^{\Delta y / 2} \int_{0}^{z_{\mathrm{I}}(-\Delta x / 2, \eta)} \rho u u \mathrm{~d} z \mathrm{~d} \eta \\
& +\int_{-\Delta y / 2}^{\Delta y / 2} \int_{0}^{z_{\mathrm{I}}(\Delta x / 2, \eta)} \rho u u \mathrm{~d} z \mathrm{~d} \eta \\
& -\int_{-\Delta x / 2}^{\Delta x / 2} \int_{0}^{z_{\mathrm{I}}(\xi,-\Delta y / 2)} \rho u v \mathrm{~d} z \mathrm{~d} \xi \\
& +\int_{-\Delta x / 2}^{\Delta x / 2} \int_{0}^{z_{\mathrm{I}}(\xi, \Delta y / 2)} \rho u v \mathrm{~d} z \mathrm{~d} \xi
\end{aligned}
$$

The Leibniz rule applied to the first term on the right hand side of Eq. (5), denoted with Term 1, produces:

$$
\begin{aligned}
\text { Term } 1= & \int_{-\Delta x / 2}^{\Delta x / 2} \int_{-\Delta y / 2}^{\Delta y / 2} \frac{\partial}{\partial t} \int_{0}^{z_{\mathrm{I}}(\xi, \eta)} \rho u \mathrm{~d} z \mathrm{~d} \eta \mathrm{d} \xi \\
& -\int_{-\Delta x / 2}^{\Delta x / 2} \int_{-\Delta y / 2}^{\Delta y / 2} \rho_{\mathrm{I}} u_{\mathrm{I}} \frac{\partial z_{\mathrm{I}}}{\partial t} \mathrm{~d} \eta \mathrm{d} \xi
\end{aligned}
$$

Due to the kinematic condition for the surface $S_{\text {I }}$ the second term on the right hand side of Eq. (6) cancels with the Term 2 in Eq. (5). Term 3 on the right hand side of Eq. (5) will be denoted with $-E_{u}$, where $E_{u}$ represents the net $x$-momentum flux that enters the current through its interface over the entire plan area of the control volume, $\Delta x, \Delta y$. Furthermore, all integrals of quantities over the current depth (i.e. between 0 and $z_{\mathrm{I}}$ ) are replaced with the product of depth and the depth-averaged quantity (according to Eq. (1)). The result is:

$$
\begin{aligned}
R M= & \int_{-\Delta x / 2}^{\Delta x / 2} \int_{-\Delta y / 2}^{\Delta y / 2} \frac{\partial\langle\rho u\rangle_{h} h}{\partial t} \mathrm{~d} \eta \mathrm{d} \xi-E_{u} \\
& -\left.\int_{-\Delta y / 2}^{\Delta y / 2}\langle\rho u u\rangle_{h} h\right|_{\xi=-\Delta x / 2} \mathrm{~d} \eta \\
& +\left.\int_{-\Delta y / 2}^{\Delta y / 2}\langle\rho u u\rangle_{h} h\right|_{\xi=\Delta x / 2} \mathrm{~d} \eta \\
& -\left.\int_{-\Delta x / 2}^{\Delta x / 2}\langle\rho u v\rangle_{h} h\right|_{\eta=-\Delta y / 2} \mathrm{~d} \xi \\
& +\left.\int_{-\Delta x / 2}^{\Delta x / 2}\langle\rho u v\rangle_{h} h\right|_{\eta=\Delta y / 2} \mathrm{~d} \xi
\end{aligned}
$$

where the symbols for "value at", e.g. $\left.\right|_{\xi=-\Delta x / 2}$, apply to all terms within integrals.

\subsection{Gravity term $G$}

The gravity term for the control volume covering the current height becomes:

$$
\begin{aligned}
G & =\int_{-\Delta x / 2}^{\Delta x / 2} \int_{-\Delta y / 2}^{\Delta y / 2} \int_{0}^{z_{\mathrm{I}}(\xi, \eta)}\left(\rho-\rho_{0}\right) g_{x} \mathrm{~d} z \mathrm{~d} \eta \mathrm{d} \xi \\
& =\int_{-\Delta x / 2}^{\Delta x / 2} \int_{-\Delta y / 2}^{\Delta y / 2}\left\langle\rho-\rho_{0}\right\rangle_{h} h g_{x} \mathrm{~d} \eta \mathrm{d} \xi
\end{aligned}
$$

\subsection{Viscous stress term $T$}

The viscous stress term is non-zero along all surfaces enclosing the control volume, so the total force due to the viscous stress is the sum of forces acting on the bottom, the interface, and the 
four bed-normal surfaces:

$$
\begin{aligned}
T= & -\int_{-\Delta x / 2}^{\Delta x / 2} \int_{-\Delta y / 2}^{\Delta y / 2} \tau_{B x} \mathrm{~d} \eta \mathrm{d} \xi+\int_{S_{\mathrm{I}}} \tau_{i x} n_{i} \mathrm{~d} S_{\mathrm{I}} \\
& -\int_{-\Delta y / 2}^{\Delta y / 2} \int_{0}^{z_{\mathrm{I}}(-\Delta x / 2, \eta)} \tau_{x x} \mathrm{~d} z \mathrm{~d} \eta+\int_{-\Delta y / 2}^{\Delta y / 2} \int_{0}^{z_{\mathrm{I}}(\Delta x / 2, \eta)} \tau_{x x} \mathrm{~d} z \mathrm{~d} \eta \\
& -\int_{-\Delta x / 2}^{\Delta x / 2} \int_{0}^{z_{\mathrm{I}}(\xi,-\Delta y / 2)} \tau_{y x} \mathrm{~d} z \mathrm{~d} \xi+\int_{-\Delta x / 2}^{\Delta x / 2} \int_{0}^{z_{\mathrm{I}}(\xi, \Delta y / 2)} \tau_{y x} \mathrm{~d} z \mathrm{~d} \xi \\
= & -\int_{-\Delta x / 2}^{\Delta x / 2} \int_{-\Delta y / 2}^{\Delta y / 2} \tau_{B x} \mathrm{~d} \eta \mathrm{d} \xi+\int_{S_{\mathrm{I}}} \tau_{i x} n_{i} \mathrm{~d} S_{\mathrm{I}} \\
& -\left.\int_{-\Delta y / 2}^{\Delta y / 2}\left\langle\tau_{x x}\right\rangle_{h} h\right|_{\xi=-\Delta x / 2} \mathrm{~d} \eta+\left.\int_{-\Delta y / 2}^{\Delta y / 2}\left\langle\tau_{x x}\right\rangle_{h} h\right|_{\xi=\Delta x / 2} \mathrm{~d} \eta \\
& -\left.\int_{-\Delta x / 2}^{\Delta x / 2}\left\langle\tau_{y x}\right\rangle_{h} h\right|_{\eta=-\Delta y / 2} \mathrm{~d} \xi+\left.\int_{-\Delta x / 2}^{\Delta x / 2}\left\langle\tau_{y x}\right\rangle_{h} h\right|_{\eta=\Delta y / 2} \mathrm{~d} \xi
\end{aligned}
$$

It should be noted that this note considers only flat bed, so that the shear stress acting on the fluid across the bed surface, $\tau_{B x}$, is equal to the viscous stress. Extrapolation to the case of rough bed, where the bed shear stress is the sum of the viscous stress and all pressure forces acting on the grains per unit area (i.e. it is due to both viscous drag and form drag) is straightforward (see Pokrajac, 2013 for details).
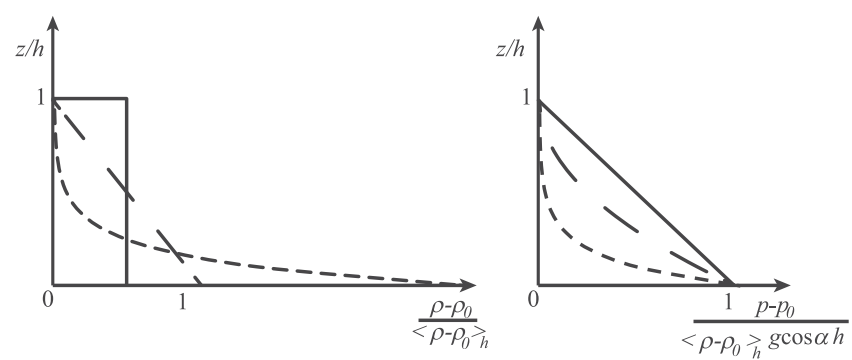

Figure 2 Shapes of density and pressure profiles for: constant (continuous line), linear (dashed line), and exponential (dotted line) density profiles

\subsection{Pressure term $P$}

The pressure term is assumed to be zero along the interface (where $p-p_{0}=0$ ) and along the bed and the two lateral bed-normal surfaces $(\eta=-\Delta y / 2$ and $\eta=-\Delta y / 2)$ where the $x$-component of the unit normal vector of the surface is zero. The remaining non-zero contributions are:

$$
\begin{aligned}
P= & \int_{-\Delta y / 2}^{\Delta y / 2} \int_{0}^{z_{\mathrm{I}}(-\Delta x / 2, \eta)}\left(p-p_{0}\right) \mathrm{d} z \mathrm{~d} \eta \\
& -\int_{-\Delta y / 2}^{\Delta y / 2} \int_{0}^{z_{\mathrm{I}}(\Delta x / 2, \eta)}\left(p-p_{0}\right) \mathrm{d} z \mathrm{~d} \eta
\end{aligned}
$$

Pressure distribution is assumed hydrostatic, so the expression for $p-p_{0}$ at a level $z$ is found by integrating the $z$-momentum equation between $z$ and $z_{\mathrm{I}}$, as:

$$
p-p_{0}=\int_{z}^{z_{\mathrm{I}}}\left(\rho-\rho_{0}\right) g \cos \alpha \mathrm{d} z
$$

A pressure coefficient, $a_{p}$, can now be defined as the force due to pressure per unit width, normalized with the force that corresponds to the constant density i.e.:

$$
a_{p}=\frac{\int_{0}^{z_{1}}\left(p-p_{0}\right) \mathrm{d} z}{\frac{1}{2}\left\langle\rho-\rho_{0}\right\rangle_{h} g \cos \alpha h^{2}}=\frac{\int_{0}^{z_{1}} \int_{z}^{z_{1}}\left(\rho-\rho_{0}\right) \mathrm{d} z \mathrm{~d} z}{\frac{1}{2}\left\langle\rho-\rho_{0}\right\rangle_{h} h^{2}}
$$

or, alternatively, using a non-dimensional coordinate $\zeta=z / h$, as:

$$
a_{p}=2 \int_{0}^{1} \frac{p-p_{0}}{\left\langle\rho-\rho_{0}\right\rangle_{h} g \cos \alpha h} \mathrm{~d} \zeta=2 \int_{0}^{1} \int_{\zeta}^{1} \frac{\rho-\rho_{0}}{\left\langle\rho-\rho_{0}\right\rangle_{h}} \mathrm{~d} \zeta \mathrm{d} \zeta
$$

The net force resulting from the pressure is now expressed as:

$$
\begin{aligned}
P= & \left.\frac{1}{2} \int_{-\Delta y / 2}^{\Delta y / 2} a_{p}\left\langle\rho-\rho_{0}\right\rangle_{h} h^{2}\right|_{\xi=-\Delta x / 2} \mathrm{~d} \eta \\
& -\left.\frac{1}{2} \int_{-\Delta y / 2}^{\Delta y / 2} a_{p}\left\langle\rho-\rho_{0}\right\rangle_{h} h^{2}\right|_{\xi=\Delta x / 2} \mathrm{~d} \eta
\end{aligned}
$$

For some simple density profiles such as those shown in Fig. 2, the integrals in Eqs (11) and (12) can be expressed analytically.

Table 1 Pressure distribution and coefficients for typical density profiles. For exponential profile $C=1-\mathrm{e}^{-\gamma}-\gamma \mathrm{e}^{-\gamma}$, where $\gamma$ is an empirical coefficient. In Altinakar et al. (1996) $\gamma$ takes values in the range (2.29-2.74)

\begin{tabular}{lccc}
\hline Profile type & $\frac{\rho-\rho_{0}}{\left\langle\rho-\rho_{0}\right\rangle_{h}}$ & $\frac{p-p_{0}}{\left\langle\rho-\rho_{0}\right\rangle_{h} g \cos \alpha h}$ & $a_{p}$ \\
\hline Constant & 1 & $1-\frac{z}{h}$ & 1 \\
Linear & $2\left(1-\frac{z}{h}\right)$ & $\left(1-\frac{z}{h}\right)^{2}$ & $\frac{2}{3}$ \\
Exponential & $\frac{\gamma}{C}\left(\mathrm{e}^{-\gamma(z / h)}-\mathrm{e}^{-\gamma}\right)$ & $\frac{1}{C}\left(\mathrm{e}^{-\gamma(z / h)}+\gamma \frac{z}{h} \mathrm{e}^{-\gamma}+C-1\right)$ & $\frac{2}{\gamma}-\frac{\gamma}{C} \mathrm{e}^{-\gamma}$ \\
\hline
\end{tabular}


These expressions are listed in Table 1 for constant, linear and exponential density profiles. The dimensional exponential profile was expressed as $\rho-\rho_{0}=A\left(\mathrm{e}^{-\gamma z / h}-\mathrm{e}^{-\gamma}\right)$, adopted from Altinakar et al. (1996), and slightly modified to ensure that at the top of the current $\rho-\rho_{0}=0$.

Figure 2 also shows the pressure profiles that correspond to the analysed density profiles. It is clear that the area of the pressure diagram is smaller for a variable density than for the corresponding constant density (equal to its depth-average). The force due to pressure is therefore smaller for the variable density, hence resulting in the pressure coefficient smaller then unity. This means that omitting pressure coefficient $a_{p}$ results in overestimating the force due to pressure gradient by factor $\left(1-a_{p}\right)$, i.e. $33 \%$ for the linear density profile. For the range of $\gamma$ values reported in Altinakar et al. (1996) (2.29-2.74) the pressure coefficient $a_{p}$ takes the values in the range $0.50-0.53$, so the pressure term is overestimated by up to $50 \%$.

\section{Differential form of momentum equation}

All previously derived terms in momentum equation are grouped, the equation is divided by $\Delta x \Delta y$, and $\Delta x, \Delta y$ are made infinitely small to yield:

$$
\begin{aligned}
& \frac{\partial\langle\rho u\rangle_{h} h}{\partial t}+\frac{\partial\langle\rho u u\rangle_{h} h}{\partial x}+\frac{\partial\langle\rho u v\rangle_{h} h}{\partial y}-e_{u} \\
& =\left\langle\rho-\rho_{0}\right\rangle_{h} h g_{x}+\frac{1}{2} g \frac{\partial a_{p}\left\langle\rho-\rho_{0}\right\rangle_{h} h^{2}}{\partial x} \cos \alpha-\tau_{\mathrm{B} x}+\tau_{\mathrm{I} x} \\
& \quad+\frac{\partial\left\langle\tau_{x x}\right\rangle_{h} h}{\partial x}+\frac{\partial\left\langle\tau_{y x}\right\rangle_{h} h}{\partial y}
\end{aligned}
$$

where $e_{u}$ is the flux of $x$-momentum entrained through the interface with the ambient fluid per unit plan area of the current. The equation for the $y$-momentum is derived in an analogous way and its final form is:

$$
\begin{aligned}
& \frac{\partial\langle\rho v\rangle_{h} h}{\partial t}+\frac{\partial\langle\rho v v\rangle_{h} h}{\partial y}+\frac{\partial\langle\rho v u\rangle_{h} h}{\partial x}-e_{v} \\
& =\left\langle\rho-\rho_{0}\right\rangle_{h} h g_{y}+\frac{1}{2} g \frac{\partial a_{p}\left\langle\rho-\rho_{0}\right\rangle_{h} h^{2}}{\partial y} \cos \beta-\tau_{\mathrm{B} y}+\tau_{\mathrm{I} y} \\
& \quad+\frac{\partial\left\langle\tau_{y y}\right\rangle_{h} h}{\partial y}+\frac{\partial\left\langle\tau_{x y}\right\rangle_{h} h}{\partial x}
\end{aligned}
$$

The $x$ and $y$-momentum equations explicitly contain only the pressure coefficient in the pressure gradient term. Other coefficients that arise from the correlations of the shape of density and velocity profiles are "hidden" in the averages of double and triple products. One of them its the well-known Boussinesq coefficient which accounts for non-uniformity of velocity profile. Expressing and analysing other terms will be the subject of further investigation.

\section{Discussion}

In order to further assess the effect of the pressure coefficient we consider a horizontal unidirectional flow in $x$ direction, and assume that: Boussinesq momentum coefficient is 1 , there is no correlation between density and velocity profiles, density profile does not change in time and space, and all shear stress terms, as well as the entrainment term are negligible. Under these assumptions Eq. (15) becomes very similar to the traditional shallow water momentum equation:

$$
\frac{\partial\langle u\rangle_{h} h}{\partial t}+\frac{\partial\langle u\rangle_{h}\langle u\rangle_{h} h}{\partial x}=a_{p} \frac{\left\langle\rho-\rho_{0}\right\rangle_{h}}{\langle\rho\rangle} g h \frac{\partial h}{\partial x}
$$

Combining Eq. (17) with the corresponding SW continuity equation:

$$
\frac{\partial h}{\partial t}+\frac{\partial\langle u\rangle_{h} h}{\partial x}=0
$$

yields the celerity (i.e. the speed of small disturbances in a current) equal to $2 \sqrt{g^{\prime} h}$, where reduced gravity is defined as:

$$
g^{\prime}=a_{p} \frac{\left\langle\rho-\rho_{0}\right\rangle_{h}}{\langle\rho\rangle_{h}} g
$$

This definition of the reduced gravity differs from the traditional one by factor $a_{p}$. This means that taking into account non-uniformity of density profile modifies the celerity by factor $\sqrt{a_{p}}$. For linear density profiles this factor is equal to 0.82 . In other words for two gravity currents with the same depth and depth-averaged density, but different density profiles, small disturbances will move $18 \%$ slower in the current with linear density profile, compared to the one with constant profile. This clearly has implications for numerical models based on SWEs: for currents with $a_{p}<1$, models which do not incorporate the pressure coefficient will overestimate celerity by factor $1 / \sqrt{a_{p}}$, i.e. by $22 \%$ and around $40 \%$ for linear end exponential density profiles, respectively.

It should be noted that the modified definition of $g^{\prime}$ given by (19) should be also applied to Froude number defined in terms of the reduced gravity.

The significance of the pressure coefficient $a_{p}$ and all associated quantities depends on the degree of non-uniformity of the density profile which in turn depends on the current composition (Altinakar et al., 1996; Kneller \& Buckee, 2000; Stagnaro $\&$ Bolla, 2014) and, arguably and non-consensually, on Froude number (Sequeiros et al., 2010; Stagnaro \& Bolla, 2014).

\section{Conclusions}

This note has presented a rigorous derivation of a 2D depthaveraged momentum equation for gravity currents for a control volume that extends between the bed and the top of the current. Equations contain a coefficient in the pressure gradient term, $a_{p}$, 
accounting for the bed-normal variation of the current density. This coefficient, named pressure coefficient, should be incorporated in SWE-based models of density currents and set to unity only when justified.

For the case of a linear density profile the pressure coefficient is 0.67 , whereas for the analysed exponential profile it is around 0.5 . This means that neglecting linear or exponential bed-normal variation of density leads to overestimation of pressure gradients by $33 \%$ and $50 \%$, respectively.

The pressure coefficient has also been incorporated in the definition of the reduced gravity for currents with non-constant density profiles: the traditional expression for $g^{\prime}$ is multiplied by factor $a_{p}$. This changes the celerity of the current by factor $\sqrt{a_{p}}$ implying that, for currents with $a_{p}<1$, celerity is overestimated in the numerical models based on traditional SWEs which do not contain the pressure coefficient.

\section{Funding}

The second author is funded by the Swiss National Science Foundation [grant number 200021_159249].

\section{Notations}

$a_{p}=$ pressure coefficient (-)

$E_{u}=$ the net $x$-momentum flux that enters the current through the interface, over the entire plan area of the control volume $\left(\mathrm{kg} \mathrm{m} \mathrm{s}^{-2}\right)$

$e_{u}=$ the net $x$-momentum flux that enters the current through the interface, per unit plan area of the current $\left(\mathrm{kg} \mathrm{m}^{-1} \mathrm{~s}^{-2}\right)$

$e_{v}=$ the net $y$-momentum flux that enters the current through the interface, per unit plan area of the current $\left(\mathrm{kg} \mathrm{m}^{-1} \mathrm{~s}^{-2}\right)$

$g^{\prime}=$ reduced gravity $\left(\mathrm{m} \mathrm{s}^{-2}\right)$

$g_{j}=$ gravity acceleration in the $j^{\text {th }}$ direction $\left(\mathrm{m} \mathrm{s}^{-2}\right)$

$g_{x}=$ gravity acceleration in the $x$ direction $\left(\mathrm{m} \mathrm{s}^{-2}\right)$

$h=$ current depth $(\mathrm{m})$

$i=$ counter, indicating the direction of any coordinate (-)

$j=$ counter, indicating the primary direction of the momentum balance equation (-)

$n_{i}=i^{\text {th }}$ component of the unit normal vector for surface $S$ pointing out of the control volume (-)

$p=$ pressure $(\mathrm{Pa})$

$p_{0}=$ ambient fluid pressure $(\mathrm{Pa})$

$S=$ surface that encloses the control volume $\left(\mathrm{m}^{2}\right)$

$S_{\mathrm{I}}=$ part of $S$ which is the interface between the current and the ambient fluid $\left(\mathrm{m}^{2}\right)$

$t=$ time (s)

$U=x$ component of the interface velocity $\left(\mathrm{m} \mathrm{s}^{-1}\right)$

$U_{i}=i^{\text {th }}$ component of the interface velocity, $i=1,2,3$ $(\equiv U, V, W)\left(\mathrm{m} \mathrm{s}^{-1}\right)$

$u=x$ component of the instantaneous fluid velocity at a point $\left(\mathrm{m} \mathrm{s}^{-1}\right)$ $u_{i}=i^{\text {th }}$ component of the fluid velocity, $i=1,2,3$ $(\equiv u, v, w)\left(\mathrm{m} \mathrm{s}^{-1}\right)$

$V=y$ component of the interface velocity $\left(\mathrm{m} \mathrm{s}^{-1}\right)$

$v=y$ component of the instantaneous fluid velocity at a point $\left(\mathrm{m} \mathrm{s}^{-1}\right)$

$W=z$ component of the interface velocity $\left(\mathrm{m} \mathrm{s}^{-1}\right)$

$w=z$ component of the instantaneous fluid velocity at a point $\left(\mathrm{m} \mathrm{s}^{-1}\right)$

$x=$ longitudinal Cartesian coordinate $(\mathrm{m})$

$x_{i}=$ Cartesian coordinate in $i^{\text {th }}$ direction, $i=1,2,3$ $(\equiv x, y, z)(\mathrm{m})$

$y=$ lateral Cartesian coordinate $(\mathrm{m})$

$z \quad=$ bed-normal Cartesian coordinate $(\mathrm{m})$

$z_{\mathrm{I}}=z$ coordinate (height above the bed) of the interface $(\mathrm{m})$

$\alpha=$ bed slope in $x$ direction (RAD)

$\beta=$ bed slope in $y$ direction (RAD)

$\gamma=$ empirical coefficient in equation for density profile (-)

$\Delta x=$ control volume length $(\mathrm{m})$

$\Delta y=$ control volume width (m)

$\eta=$ lateral coordinate of the local coordinate system centered at an arbitrary $x, y$ point $(\mathrm{m})$

$\vartheta_{i}=i^{\text {th }}$ component of the velocity of the surface $S\left(\mathrm{~m} \mathrm{~s}^{-1}\right)$

$\xi=$ longitudinal coordinate of the local coordinate system centered at an arbitrary $x, y$ point (m)

$\rho=$ current density $\left(\mathrm{kg} \mathrm{m}^{-3}\right)$

$\rho_{0}=$ ambient fluid density $\left(\mathrm{kg} \mathrm{m}^{-3}\right)$

$\tau_{\mathrm{B} x}=x$ component of the bed shear stress, i.e. $\tau_{x z}$ acting at the bed level $(\mathrm{Pa})$

$\tau_{\mathrm{B} y}=y$ component of the bed shear stress, i.e. $\tau_{y z}$ acting at the bed level $(\mathrm{Pa})$

$\tau_{\mathrm{I} x}=x$ component of the shear stress at the interface between the current and the ambient fluid, i.e. $\tau_{x z}$ acting at the interface $(\mathrm{Pa})$

$\tau_{\mathrm{I} y}=y$ component of the shear stress at the interface between the current and the ambient fluid, i.e. $\tau_{y z}$ acting at the interface $(\mathrm{Pa})$

$\tau_{i j}=i, j$ component of viscous stress $(\mathrm{Pa})$

$\psi=$ general fluid variable

$\forall \quad=$ control volume $\left(\mathrm{m}^{3}\right)$

\section{References}

Adduce, C., Sciortino, G., \& Proietti, S. (2012). Gravity currents produced by lock exchanges: Experiments and simulations with a two-layer shallow-water model with entrainment. Journal of Hydraulic Engineering, 138(2), 111-121.

Altinakar, M. S., Graf, W. H., \& Hopfinger, E. J. (1996). Flow structure in turbidity currents. Journal of Hydraulic Research, 34(5), 713-718.

Chu, F. H., Pilkey, W. D., \& Pilkey, O. H. (1979). An analytical study of turbidity current steady flow. Marine Geology, 33(3-4), 205-220.

Ellison, T. H., \& Turner, J. S. (1959). Turbulent entrainment in stratified flows. Journal of Fluid Mechanics, 6(03), 423-448. 
Hogg, A. J., \& Pritchard, D. (2004). The effects of hydraulic resistance on dam-break and other shallow inertial flows. Journal of Fluid Mechanics, 501, 179-212.

Kneller, B. C., Bennett, S. J., \& McCaffrey, W. D. (1999). Velocity structure, turbulence and fluid stresses in experimental gravity currents. Journal of Geophysical Research: Oceans, 104(C3), 5381-5391.

Kneller, B., \& Buckee, C. (2000). The structure and fluid mechanics of turbidity currents: A review of some recent studies and their geological implications. Sedimentology, 47, 62-94.

Lombardi, V., Adduce, C., Sciortino, G., \& La Rocca, M. (2015). Gravity currents flowing upslope: Laboratory experiments and shallow-water simulations. Physics of Fluids, 27(1), 016602.

Ottolenghi, L., Adduce, C., Inghilesi, R., Armenio, V., \& Roman, F. (2016a). Entrainment and mixing in unsteady gravity currents. Journal of Hydraulic Research, 54(5), 541-557.

Ottolenghi, L., Adduce, C., Inghilesi, R., Roman, F., \& Armenio, V. (2016b). Mixing in lock-release gravity currents propagating up a slope. Physics of Fluids, 28(5), 056604.
Parker, G., Garcia, M., Fukushima, Y., \& Yu, W. (1987). Experiments on turbidity currents over an erodible bed. Journal of Hydraulic Research, 25(1), 123-147.

Pokrajac, D., \& Kikkert, G. A. (2011). RADINS equations for aerated shallow water flows over rough beds. Journal of Hydraulic Research, 49(5), 630-638.

Sequeiros, O. E., Spinewine, B., Beaubouef, R. T., Sun, T., García, M. H., \& Parker, G. (2010). Characteristics of velocity and excess density profiles of saline underflows and turbidity currents flowing over a mobile bed. Journal of Hydraulic Engineering, 136(7), 412-433.

Stacey, M. W., \& Bowen, A. J. (1988). The vertical structure of density and turbidity currents: Theory and observations. Journal of Geophysical Research: Oceans, 93(C4), 35283542.

Stagnaro, M., \& Bolla Pittaluga, M. (2014). Velocity and concentration profiles of saline and turbidity currents flowing in a straight channel under quasi-uniform conditions. Earth Surface Dynamics, 2(1), 167-180.

Ungarish, M. (2009). An introduction to gravity currents and intrusions. New York: CRC Press. 